\title{
Simultaneous Kidney and Liver Resection in Renal Cell Carcinoma Liver Metastases: A Tale of Two Cases
}

\author{
Indah Jamtani ${ }^{1}$, Adianto Nugroho* ${ }^{1}$, Syamsu Hudaya ${ }^{2}$, Rofi Y. Saunar ${ }^{1}$, Aditomo Widarso ${ }^{1}$, \\ Taslim Poniman ${ }^{1}$ \\ ${ }^{1}$ Digestive Division, Department of Surgery, Fatmawati Central General Hospital, Jakarta, Indonesia \\ 2 Urology Division, Department of Surgery, Fatmawati Central General Hospital, Jakarta, Indonesia
}

\section{ARTICLE INFO}

Received : 10 August 2020

Reviewed : 04 September 2020

Accepted : 22 October 2020

\section{Keywords:}

liver metastasis, renal cell carcinoma, simultaneous kidney liver resection

\author{
${ }^{*}$ Corresponding author: \\ Adianto Nugroho \\ Fatmawati Central General Hospital \\ Digestive Division, Department \\ of Surgery, Fatmawati Central \\ General Hospital, Jakarta, Indonesia \\ adiyusuf97@gmail.com
}

\author{
A BSTRACT
}

\begin{abstract}
Introduction: Resection of renal cell carcinoma liver metastases (RCCLM) is known to provide a good survival outcome. Simultaneous RCCLM resection with the primary tumor is commonly done in cases with direct extension of locally advanced RCC to liver parenchyma. Cases of safe simultaneous resection of liver and kidney in malignancy have not been reported before in Indonesia.
\end{abstract}

\begin{abstract}
Case Presentation: We aim to describe two cases of left-sided RCC with right-sided RCCLM who underwent simultaneous kidney and liver resection. Both patients had good short-term survival with no intra-operative and post-operative complications.
\end{abstract}

Conclusion: Although simultaneous kidney and liver resection poses challenges of substantial morbidity, a safe surgical and multidisciplinary approach could be implemented in selected cases with a good surgical outcome.

\section{INTRODUCTION}

The advancement in surgical skills along with improvement in anesthesia and perioperative management played important role in the progress of safe liver surgery. The current morbidity of liver surgery in high-volume, specialized centers comes close to $15 \%$ $50 \%$ and mortality $<5 \%$ [1]. This evolvement has led to a rise of indications for hepatic resections of metastatic diseases. Liver tumors secondary to metastatic kidney malignancies represent about $5-8 \%$ of non-colorectal non-endocrine liver metastases and are present in $20 \%$ of metastatic kidney cancers [2,3]. Resection of renal cell carcinoma liver metastases (RCCLM) has been gaining more interest and is increasingly being performed as a common surgical procedure [1,2]. Simultaneous resection of RCCLM with the primary tumor has been reported before, most commonly in cases with direct extension of RCC into the adjacent liver parenchyma. Safe liver resections have only been developed more in the past five to ten years in Indonesia, let alone a simultaneous resection with nephrectomy. Each procedure has its high morbidity and postoperative mortality rate. Therefore, a combination of a multidisciplinary approach and highly specialized surgical technique is key in successful surgery and management of these high-stakes' cases.

Here, we report two cases of simultaneous kidney and liver resection for left-sided RCC with metastases to the right liver. To our knowledge, this is the first report of its kind in our region.

\section{CASE PRESENTATION}

The first case is a 17-year-old male patient with stage IV left-sided RCC and synchronous liver metastases. Upon computed tomography (CT) scan, the size of left-sided RCC was $15 \times 12 \times 10 \mathrm{~cm}$ and the RCCLM was $3 \times 3 \times 2 \mathrm{~cm}$ in segment 5 of the liver (Figure 1). The patient was in optimal condition with an ECOG score of 0 and normal pre-operative lab values. Simultaneous resection of the RCCLM along with the primary tumor was discussed and decided. Intra-operatively left side nephrectomy was performed first by a urologist, followed by the right anterior sectionectomy of the liver by a hepatobiliary pancreatic surgeon. Intra-operative blood loss was $500 \mathrm{cc}$. The patient was in the intensive care unit (ICU) for two days following surgery and was discharged at postoperative day (POD) 10 without any perioperative complication. 

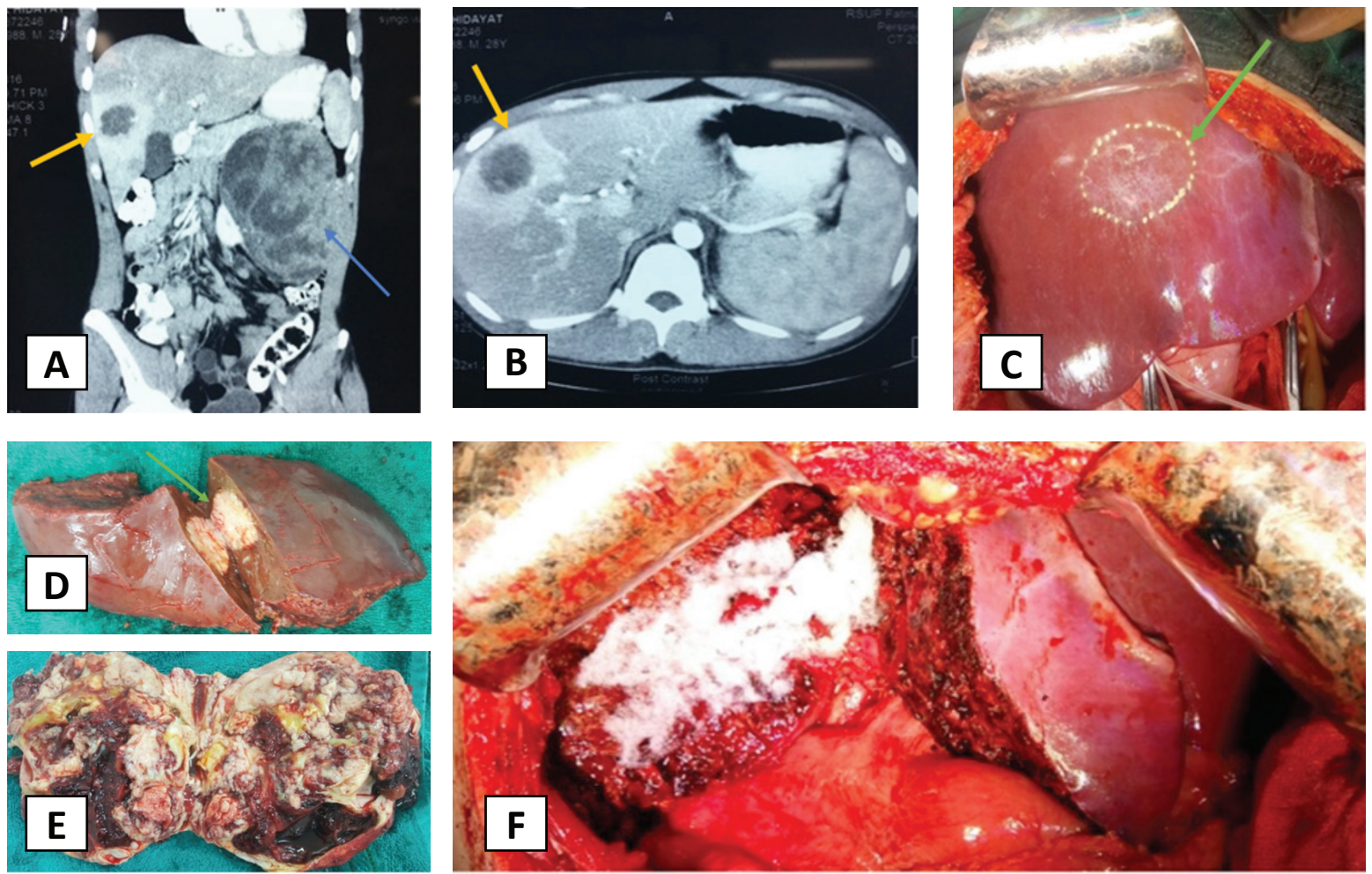

Figure 1. Case 1. Male, 17 years old, Left RCC, S5 Liver Metastases underwent Left radical nephrectomy and right anterior; A. and B. show pre-operative CT scans: blue arrow shows primary tumor, left-sided RCC and yellow arrow shows segment 5 liver metastases; C. intraoperative LM, D. resected right anterior liver segment including RCCLM, green arrow shows the margin of the metastases; E. left kidney and primary tumor specimen; F. liver surface after resection.
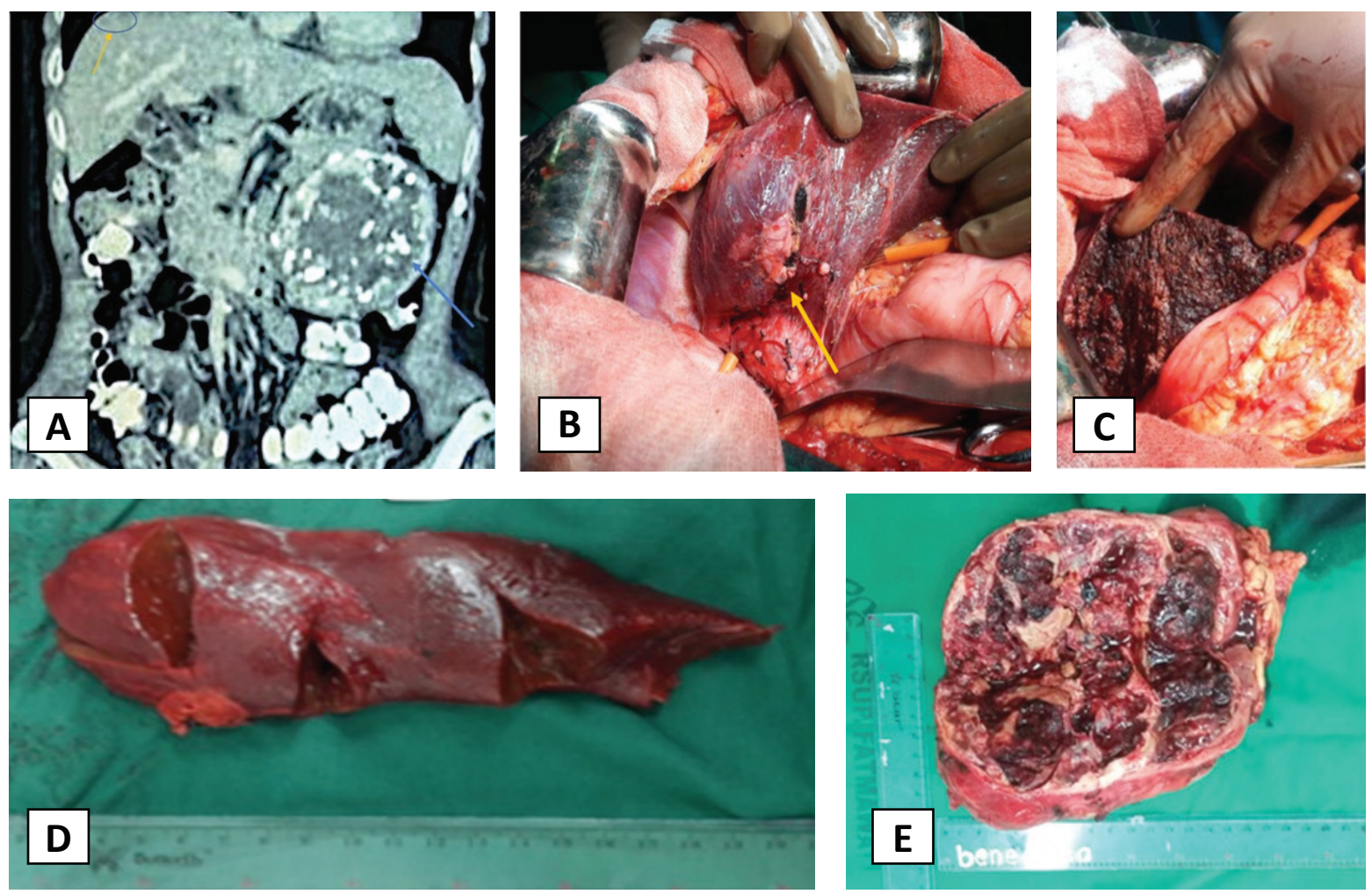

Figure 2. Case 2: Female, 69 years old, Left RCC, S7 Liver Metastases underwent Left radical nephrectomy and right posterior sectionectomy; A. CT scan showing left RCC (blue arrow) and right-sided LM nodule (yellow arrow), B. intraoperative LM nodule, C. Liver surface post-resection, D. liver specimen, E. kidney specimen. 
The second case was a 69-year-old female patient with $7 \times 5 \times 5 \mathrm{~cm}$ stage IV left-sided RCC. She had a synchronous RCCLM in segment 7 with a size of $3 \times 2 \times 2$ $\mathrm{cm}$ (Figure 2). She was in optimal condition with an ECOG score of 0 and normal pre-operative lab values. She underwent simultaneous resection of RCCLM along with the primary tumor. Left side nephrectomy was performed first by urologists followed by the right posterior sectionectomy of the liver by a hepatobiliary pancreatic surgeon. Intra-operative blood loss was 200 cc. The patient was cared for in the ICU postoperative day one and was moved toward the following day, subsequently discharged at POD 7.

The histopathology of the primary tumor in both cases showed clear cell RCC with the same cancer cells evident in the liver nodules. Upon follow-up at the outpatient clinic, both patients had recovered well and were scheduled for systemic targeted therapy.

\section{DISCUSSION}

The constant progression in the field of liver surgery, including perioperative care, led to the increasing number of liver resection in metastatic diseases. Although for many years' resection in non-colorectal, non-endocrine liver metastases have been avoided, recently the safety and good overall and disease-free survival have been demonstrated in RCCLM resections. One of the earlier reports of RCCLM resections, in a cohort of more than 10 cases, was by a German group in 1997 which showed a bleak short-term outcome with a mortality of $31 \%$. However, the short-term and longterm overall outcomes have improved considerably since then making resection of RCCLM a safe choice of treatment $[1,4]$.

Studies from the Netherlands and United States of America (USA) described the incidence of metastases of renal cell carcinoma (RCC) in the liver is about 20$30 \%$, the third most common site after lung and bones and associated with worse overall survival (OS) and disease-free survival (DFS). Synchronous liver metastases have been reported to occur in $20-30 \%$ of all RCC cases while $20-40 \%$ of cases developed metachronous metastases after curative intent nephrectomy of the primary tumor. Synchronous liver metastases in RCC have been shown to have a worse overall survival outcome when compared to patients with metachronous $\operatorname{LM}[3,4]$. Disease-free survival of fewer than 24 months after primary curative intent was shown to have a poor prognosis [1,4]. However, data and reports on incidence, prognosis, OS, and DFS of metastatic RCC have been limited in Indonesia.

Generally, RCCLM does not occur as a single organ metastasis; only in $5 \%$ of cases is the liver the only organ affected. The prognosis of RCCLM is especially poor and significantly worse than metastases to other organs $[1,4]$. Despite the advancement of targeted therapy, an Ro surgical resection is still regarded as the most favorable approach for the long-term survival of metastatic RCC $[4,5,6]$. While curative intent of surgical intervention for pulmonary metastases of RCC has shown significant OS improvement, liver resection, or radiofrequency ablation (RFA) of RCCLM has been controversial [3,6]. However, recent studies have demonstrated a prolonged survival outcome in RCCLM treated with surgical intervention $[3,4]$.

We presented two cases of solitary synchronous RCCLM with no other metastatic location. While RO resection of RCCLM after primary curative intent nephrectomy showed favorable outcomes, the risk of liver failure in high-volume major hepatectomy is relatively high. In addition, radical nephrectomy itself has high morbidity, let alone simultaneous LM resection. Therefore, simultaneous resections were only done in selected cases with favorable prognoses based on disease stage, extrahepatic disease, and performance status. A case-by-case multidisciplinary approach evaluation is implemented in determining those selected cases. Patients with a liver limited metastatic RCC and good performance status are strong candidates for simultaneous resection as it provides a survival benefit [2]. Most commonly, simultaneous resection of LM and primary RCC tumor was done in cases with a direct extension of the tumor to liver parenchyma to achieve en bloc resection for cancer control [6].

In our study, both cases had left-sided RCC with no direct extension to surround organs and synchronous rightsided LM. While one patient was young and the other was above 60 years old, both were relatively fit with an ECOG score of 0 . The cases were discussed between the primary surgeon and other departments involved, including radiologists, hepatobiliary-pancreatic surgeons, and intensivists, preoperatively. A complete assessment of the size and extent of the primary tumor and LM were discussed by the surgeons and radiologists through radiological studies and a surgical plan of simultaneous resection of RCCLM along with the primary tumor was developed. Due to the bulky nature and large size of the tumor, an open approach instead of minimally invasive surgery was chosen. In addition, a left-sided nephrectomy was conducted first in both cases to avoid injury during nephrectomy caused by increased portal pressure after hepatectomy. Intra-operative and post-operative care of the patient was extensively evaluated and prepared by the surgeons, anesthesiologists, and intensivists to prevent adverse cataclysmic events, intra-, or post-operatively. Preparations for the need for intra- and post-operative transfusions, post-operative dialysis, and/or CRRT were carefully conducted pre-operatively, including the insertion of a short-term three-way hemodialysis catheter. 
After resection of the primary tumor, an anatomical right anterior and right posterior sectionectomy liver resection of the RCCLM was done in each case, respectively. Anatomical resection was considered as it is technically easier and faster with additional oncologic superiority than a metastasectomy or a wedge resection. The Glissonean approach was elected in both cases. After isolating the hepatoduodenal ligament and preparing for the Pringle maneuver, the Glissonean pedicle of each affected site was isolated and ligated (anterior and posterior right pedicle respectively). Parenchymal transection was done with the help of CUSA in combination with clamp-crushing in an anterior approach fashion using hanging maneuver. Selective use of Pringle maneuver was practiced. The combination of the Glissonean approach, the application of Pringle, and CUSA reduced the risk of major bleeding. The total intraoperative blood loss was $500 \mathrm{cc}$ and $200 \mathrm{cc}$ in the case with right anterior and right posterior sectionectomy, respectively.

In cases of metastatic RCC, a multidisciplinary approach consists of hepatobiliary-pancreatic surgeons, urologists, anesthesiologists, pathologists, and radiologists should always be the standard practice. Only with this approach, a specifically tailored treatment for each patient can safely proceed. Accurate patient selection, satisfactory peri-operative management, and safe surgical approaches are beneficial for short-term and long-term prognosis [2].

\section{CONCLUSIONS}

Proper patient selection and strict adherence to the basic principle of liver resection, in a multidisciplinary approach fashion, are key to a safe simultaneous kidney and liver resection.

\section{DECLARATIONS}

\section{Competing of Interest}

The author(s) declare no competing interest in this paper.

\section{REFERENCES}

1. Pikoulis E, Margonis GA, Antoniou E. Surgical management of renal cell cancer liver metastases. Scand J Surg. 2016;105(4):263-8.

2. Pinotti E, Montuori M, Giani A, et al. Surgical treatment of liver metastases from kidney cancer: a systematic review. ANZ J Surg. 2019;89(1-2):32-7.

3. Langan RC, Ripley RT, Davis JL, et al. Liver directed therapy for renal cell carcinoma. J Cancer. 2012;3:184-90.

4. Ruys AT, Tanis PJ, Nagtegaal ID, et al. Surgical treatment of renal cell cancer liver metastases: a population-based study. Ann Surg Oncol. 2011;18(7):1932-8.

5. Beetz O, Soffker R, Cammann S, et al. Extended hepatic metastasectomy for renal cell carcinoma-new aspects in times of targeted therapy: a single-center experience over three decades. Langenbecks Arch Surg. 2020;405(1):97-106.

6. Fujii $M$, Kamimura $T$, Tsukino $H$, et al. Safe resection of renal cell carcinoma with liver invasion using liver hanging technique supported by preoperative portal vein embolization. Case Rep Urol. 2018;2018:5139034.

\section{Acknowledgment}

Not applicable 